\title{
A Real-Time Dressing Method for Metal Lapping Pads Based on the Thermal-Deformation Effect
}

\section{Lingyu Zhao ( $\nabla$ zhao_lingyu@foxmail.com )}

Xi'an Jiaotong University

\section{Huiying Zhao}

Xi'an Jiaotong University

\section{Hairong Wang}

Xi'an Jiaotong University

\section{Ruiqing Xie}

Xi'an Jiaotong University

\section{Mingchen Chao}

Xi'an Jiaotong University

\section{Mingzhuang Zhang}

Research Center of Laser Fusion, China Academy of Engineering Physics

\section{Shijie Zhao}

Research Center of Laser Fusion, China Academy of Engineering Physics

\section{Research Article}

Keywords: Real-time dressing, bimetal thermal deformation effect, thermal deformation error compensation, process parameter optimization

Posted Date: September 7th, 2021

DOI: https://doi.org/10.21203/rs.3.rs-874094/v1

License: (c) (1) This work is licensed under a Creative Commons Attribution 4.0 International License. Read Full License

Version of Record: A version of this preprint was published at The International Journal of Advanced Manufacturing Technology on February 8th, 2022. See the published version at https://doi.org/10.1007/s00170-022-08869-y. 


\title{
A real-time dressing method for metal lapping pads
}

\section{based on the thermal-deformation effect}

Lingyu Zhao ${ }^{1 *}$, Huiying Zhao ${ }^{1}$, Hairong Wang ${ }^{1}$, Ruiqing Xie ${ }^{1,2}$, Mingchen Chao ${ }^{1}$, Mingzhuang Zhang ${ }^{2}$, Shijie Zhao ${ }^{2}$

* Lingyu Zhao, E-mail : zhao_lingyu@ @oxmail.com

1 State Key Laboratory for Manufacturing Systems Engineering, Xi'an Jiaotong University, Xi'an 710049, Shaanxi, China

2 Research Center of Laser Fusion, China Academy of Engineering Physics, Mianyang 621900, Sichuan, China

\begin{abstract}
The surface shape and accuracy of the metal lapping pad are important factors that affect the performance of the lapping process for flat optical components. A real-time control system for metal-lapping-pad surface shape (RCLPS) based on the bimetal thermal-deformation effect is proposed. Unlike traditional dressing methods (e.g., turning dressing with a diamond tool), real-time dressing based on the RCLPS system is a material-loss-free and in-process dressing method. A full-aperture lapping turntable based on the RCLPS system was designed, and the working heat was analyzed. A thermal-deformation model was established through regression analysis. Finally, experiments were conducted to verify the functionality of the RCLPS system. During turning dressing (turntable rotational speed $n_{m}=100 \mathrm{rpm}$ ), the thermal-deformation error caused by the working heat of the turntable-shaft system was compensated by the RCLPS system. During the lapping process for optical elements $\left(n_{m} \leq 20\right.$ $\mathrm{rpm}$ ), the real-time dressing of the lapping-pad surface shape was controlled by the coolant temperature of the RCLPS system, permitting optimization and adjustment of the process performance of the plane optical component. The effectiveness and practicality of the RCLPS system were demonstrated experimentally.
\end{abstract}

Keywords: Real-time dressing, bimetal thermal deformation effect, thermal deformation error compensation, process parameter optimization

\section{Introduction}

Plane lapping is used to produce the high-precision plane components required for integrated circuits, giant lasers, optical lenses, and other devices [1-2]. According to recent research, the evaluation criteria for lapping and polishing plane components (including material-removal rate, surface roughness, and flatness) are affected by the dressing of the lapping-and-polishing-pad surface [3-5]. Unless the lapping-and-polishing pad is continually dressed, it experiences glazing, passivation, and wear, gradually decreasing the process performance of the plane component [6]. Therefore, improving this dressing process would improve the process performance of plane components [7-8].

Use of a diamond dresser is the most traditional method of dressing a lapping pad [9-12]. However, it results in material losses, reducing the pad's service life. To avoid loss of production efficiency due to numerous pad replacements, three categories of more efficient dressing methods have been studied in recent years. First, in-process dressing methods without a diamond dresser have been considered [13]. For 
example, Chiou et al. [14] proposed a dressing method for a fixed-abrasive lapping pad based on surface coating technology, successfully improving the surface roughness of the plane components. Second, dressing methods based on new types of diamond dresser have been researched [15]. For example, an organic-composite diamond dresser was adopted by Tsai et al. to reduce the material loss of the polishing pad [16]. Finally, the self-dressing effect of lapping and polishing processes has been studied. For example, Lee. et al. pointed out that self-dressing can be realized in a fixed-abrasive lapping pad by optimizing the process parameters [17]. In summary, with a diamond dresser, material losses of the lapping-and-polishing pad cannot be avoided. Therefore, for efficiency, it is necessary to propose real-time, material-loss-free, and in-process dressing methods.

Tin lapping pads are commonly used in processing optical components such as YAG crystals [18]. Such pads are usually dressed by turning with a diamond tool at a constant speed feed. During the lapping process, the surface of the pad must be repeatedly corrected. However, turning dressing affects the service life of the pad and the production efficiency of the components. Instead, in this study, a system for real-time control of lapping-pad surface shape (RCLPS) is proposed, based on the bimetal thermal-deformation effect. It avoids lapping-pad material loss; it is an in-process and real-time dressing method that controls the metal lapping-pad surface shape through a temperature parameter $t_{c}$; and it can effectively improve the service life of tin lapping pads (and therefore component-production efficiency). Finally, in turning dressing, the thermal-deformation error due to the working heat $Q$ is compensated by the RCLPS system.

The paper is organized as follows: First, the structure and design principles of the RCLPS system are described. Second, the bimetal thermal-deformation effect is analyzed, based on the thin-plate theory from classical elasticity. A full-aperture lapping turntable with the RCLPS system is proposed, and the source of the thermal-deformation error for the lapping-pad surface is investigated. A regression-analysis method for the flexural deformation of a lapping-pad surface is proposed. Then, the dressing effect of the RCLPS system on the surface shape of metal-tin lapping pads is tested experimentally under various conditions, and orthogonal experiments are conducted to verify the influence of $t_{c}$ on process performance. We conclude that the method is effective and practical for dressing lapping-pad surfaces.

\section{Method and theory}

\subsection{Structure and design principle for the RCLPS system}

The RCLPS system is composed of a deformation plate, constraint plate, and control unit (Figure 1). The constraint and deformation plates are solid discs made of metals 1 and 2, respectively; their volumes are $\pi D_{1} \times h_{1}$ and $\pi D_{2} \times h_{2}$, where $D$ refers to diameter and $h$ to plate thickness. The control unit consists of a control unit plate with a cavity structure, circulating cooling water, and a device for water-temperature control. The control unit plate is a circular disc of volume $\pi D_{3} \times h_{3}$, made of metal 2 and containing a hollow cavity. Circulating cooling water with constant pressure $P_{0}$ passes through the cavity; its temperature $t$ is controlled by the temperature-control device. The temperature fields of the deformation plate and constraint plate are in turn controlled by the coolant temperature $t_{c}$. The design principles and control parameters of the RCLPS system are as follows:

$$
\text { Control parameter: } t_{c}
$$




$$
\text { Design principles : }\left\{\begin{array}{c}
D_{1}=D_{2}=D_{0} \\
h_{1} \leq \frac{D_{1}}{8} \\
h_{2} \leq \frac{D_{2}}{8} \\
\alpha_{1} \geq 1.5 \alpha_{2}
\end{array}\right.
$$

where $\alpha_{1}$ and $\alpha_{2}$ are the linear expansion coefficients of the constraint and deformation plates, respectively.

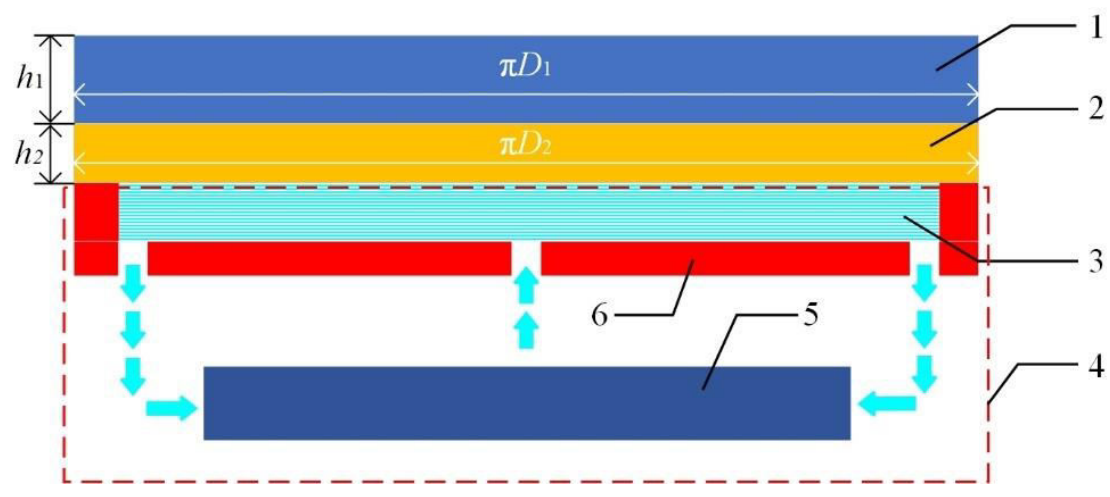

Fig. 1 Structure of the RCLPS system: 1. Constraint plate; 2. Deformation plate; 3. Circulating cooling water; 4. Control unit; 5. Temperature control device for circulating cooling water; 6 . Control unit plate

According to Eq. (2), the thickness-to-diameter ratios for the constraint and deformation plates are less than 1/8; therefore, the circular-thin-plate elastic-deformation theory is used for stress-deformation analysis. The deformation and constraint plates are made of different materials (with different linear-expansion coefficients). The different expansion rates of the two materials as the temperature-field changes result in thermal stress, which causes deformation; this phenomenon is called the bimetal thermal-deformation effect. The resulting flexure deformation of the deformation plate (which affects the surface shape of the lapping pad) is controlled by the parameter $t$.

\subsection{The bimetal thermal-deformation effect}

An example of a system that experiences the bimetal thermal-deformation effect is shown schematically in Figure 2(a), where disc 1 and 2 are rigidly connected but made from materials with different linear expansion coefficients $\alpha_{1}$ and $\alpha_{2}$. Both discs are assumed to be at the same temperature. The thermal stress generated by the different thermal expansions can be expressed as [19]

$$
\sigma_{t}=\left[E_{2} /\left(1-v_{2}\right)\right]\left(\alpha_{1}-\alpha_{2}\right)\left[T-T_{0}\right],
$$

where $\sigma_{t}$ represents the thermal stress; $E_{2}$ and $v_{2}$ are the Young's modulus and Poisson's ratio of discs 2;

$T$ is the current temperature of the discs, and $T_{0}$ is their initial temperature. 


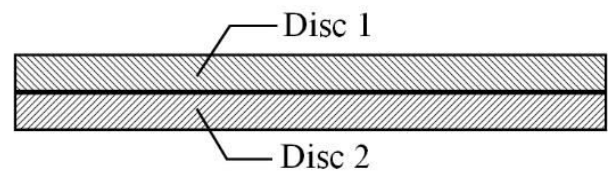

(a)

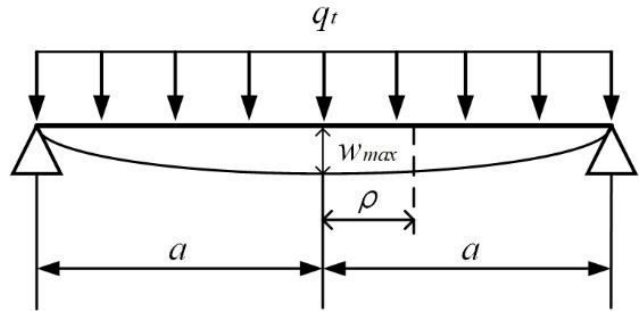

(b)

Fig. 2 Principle of the bimetal thermal-deformation effect: (a) Two discs, each composed of a different material, viewed as a single composite plate; (b) Transverse force $q_{t}$ on composite plate at radial distance $\rho$ leads to flexural deformation $w$.

According to thin-plate thermal-deformation theory [20], the flexure deformation of the thin plate is caused by the transverse force in the neutral plane. Let $q_{t}$ be the equivalent transverse force produced by $\sigma_{t}$, and $w$ be the flexural deformation for $q_{t}$. The composite plate is defined as a thin plate composed of discs 1 and 2; a simplified mechanical model is shown in Figure 2(b). The differential equation of the elastic deformation surface is

$$
D\left(\frac{d^{2}}{d \rho^{2}}+\frac{1}{\rho} \frac{d}{d \rho}\right)\left(\frac{d^{2} w}{d \rho^{2}}+\frac{1}{\rho} \frac{d w}{d \rho}\right)=q_{t},
$$

where $\rho$ represents the radial distance, and $D$ is the equivalent bending stiffness of the composite plate. Eq. (4) is an ordinary differential equation, the general solution of which is

$$
w=C_{1} \ln \rho+C_{2} \rho^{2} \ln \rho+C_{3} \rho^{2}+C_{4}+\frac{q_{t} \rho^{4}}{64 D}
$$

where $C_{1}, C_{2}, C_{3}$, and $C_{4}$ are undetermined coefficients that are solved from the boundary conditions.

The composite plate is assumed to be a simply supported edge. Therefore, the boundary conditions are

$$
\left\{\begin{array}{l}
\left.w(\rho)\right|_{\rho=a}=0 \\
\left.M(\rho)\right|_{\rho=a}=0
\end{array},\right.
$$

where $M$ is the bending moment for $q_{t}$.

From Eq. (6), $C_{1}, C_{2}, C_{3}$, and $C_{4}$ are

$$
\left\{\begin{array}{c}
C_{1}=0 \\
C_{2}=0 \\
C_{3}=-\frac{(3+\mu) q_{t} a^{2}}{32(1+\mu) D} \\
C_{4}=\frac{q_{t} a^{4}}{64 D} \frac{5+\mu}{1+\mu}
\end{array},\right.
$$

where $\mu$ and $a$ are the equivalent Poisson's ratio and radius of the composite plate, respectively. 
The curve for $w$ is obtained by inserting Eq. (7) into Eq. (5):

$$
w=\frac{q_{t} a^{4}}{64 D}\left(1-\frac{\rho^{2}}{a^{2}}\right)\left(\frac{5+\mu}{1+\mu}-\frac{\rho^{2}}{a^{2}}\right) .
$$

From Eq. (8), the maximum flexural deformation $w_{\max }$ is at $\rho=0$ :

$$
w_{\mathrm{MAX}}=\frac{q_{t} a^{4}}{64 D}\left(\frac{5+\mu}{1+\mu}\right)
$$

\subsection{Structure of full-aperture lapping turntable based on the RCLPS system}

The full-aperture (lapping pad diameter $D_{0}=1200 \mathrm{~mm}$ ) lapping turntable based on the RCLPS system consists of a metal-tin lapping pad, an RCLPS system, a cast-iron support plate, a turntable mandrel, a turntable bearing, and a torque motor (Figure 3). The lapping turntable is driven by a torque motor to rotate $360^{\circ}$ along the $C$ axis; it is supported in the axial and radial directions by high-precision ball bearings. The surface shape of the lapping pad is deterministically controlled by the RCLPS system using the temperature parameter $t_{c}$.

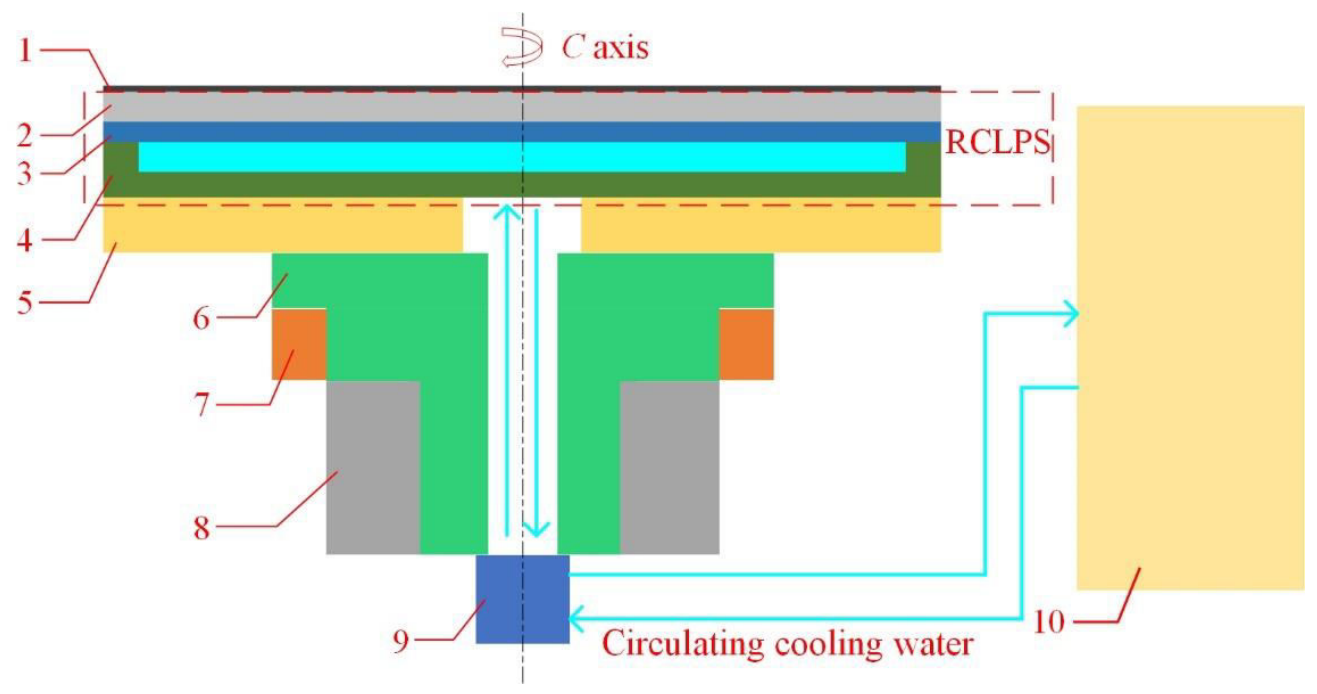

Fig. 3 Structure of the full-aperture lapping turntable based on the RCLPS system: 1. Metal-tin lapping pad; 2. Constraint plate (material: aluminum alloy); 3. Deformation plate (material: cast iron); 4. Control-unit plate (material: cast iron); 5. Support plate (material: cast iron); 6. Core shaft; 7. Turntable bearing; 8. Torque motor; 9. Rotary joint; 10. High-precision water chillers

The design parameters for the RCLPS system come from Eq. (2) and the structure of the full-aperture lapping turntable. Because the control-unit plate and the cast-iron support plate are rigidly connected, the same material (gray cast iron, $\alpha_{2}=11.1 \times 10^{-6} /{ }^{\circ} \mathrm{C}$ ) is used for them and for the deformation plate, reducing thermal stress at the connection of these three components. Aluminum alloy was selected as the material of the constraint plate; its linear-expansion coefficient, $\alpha_{1}=23.0 \times 10^{-6} /{ }^{\circ} \mathrm{C}$, satisfies Eq. (2). An epoxy-resin heat-insulation layer is used to bond the lapping and constraint plates. The coolant temperature $t_{c}$ of the RCLPS system is adjusted by the high-precision water chiller; it controls the flexural deformation of the lapping-pad surface. According to Eqs. (1) and (2), the control and design parameters of the RCLPS system for the full-aperture lapping turntable are as follows: 


$$
\text { design parameters: }\left\{\begin{array}{c}
D_{1}=D_{2}=D_{0}=1200 \mathrm{~mm} \\
h_{1}=45 \mathrm{~mm}=\frac{D_{1}}{26} \\
h_{2}=25 \mathrm{~mm}=\frac{D_{2}}{48} \\
\alpha_{1}=2 \alpha_{2}, \alpha_{1}=23 \times 10^{-6} /{ }^{\circ} \mathrm{C}, \alpha_{2}=11.1 \times 10^{-6} /{ }^{\circ} \mathrm{C}
\end{array}\right.
$$

The RCLPS system is symmetrical with respect to the $C$-axis.

\subsection{Source analysis of thermal-deformation error for the full-aperture lapping turntable}

The thermal-deformation error is caused by the working heat $Q$ of the machine tool. The full-aperture lapping turntable based on the RCLPS system uses high-precision ball bearings as supporting parts and is directly driven by a torque motor. Therefore, $Q$ is mainly the electromagnetic and frictional heat generated by the motor and the turntable bearing:

$$
Q=Q_{f}+Q_{e},
$$

where $Q_{f}$ and $Q_{e}$ are the friction heat and electromagnetic heat generated by the bearing and the torque motor, respectively.

According to an empirical formula [21], the frictional heat of the ball bearings is

$$
Q_{f}=1.047 \times 10^{-4} n M \text {, }
$$

where $n$ represents the rotational speed of the bearing inner ring and $M$ is the frictional torque of the bearings. Applying the direct-drive method to the turntable, the bearing inner-ring speed $n$ can be expressed as

$$
n=n_{m},
$$

where $n_{m}$ is the rotational speed of the torque motor.

The frictional torque $M$ is due to both the external load and the viscous friction of the bearing. Therefore, $M$ is

$$
M=M_{o}+M_{l},
$$

where $M_{0}$ and $M_{l}$ are the external-load torque and viscous-friction torque, respectively. The external-load torque $M_{0}$ is

$$
M_{0}=f_{0} k_{0} D_{m},
$$


where $f_{0}$ is a coefficient determined by the bearing structure, $k_{0}$ represents the equivalent load, and $D_{m}$ is the pitch-circle diameter of the ball bearing. The viscous-friction torque $M_{l}$ is

$$
M_{1}=\left\{\begin{array}{ll}
10^{-7} f_{1}(v n)^{2 / 3} D_{m}{ }^{3} & v n \geq 2000 \\
160 \times 10^{-7} f_{1} D_{m}{ }^{3} & v n<2000
\end{array}\right. \text {, }
$$

where $f_{l}$ is the structure coefficient of the bearing, which is determined by the design and lubrication, and $v$ represents the kinematic viscosity, which is determined by the lubricant.

The thermal load of the torque motor mainly originates from electromagnetic heat. According to an empirical formula [22], the electromagnetic heat $Q_{\mathrm{e}}$ of the torque motor is

$$
Q_{e}=P_{i n} \times\left(1-\eta_{m}\right)
$$

where $P_{i n}$ is the input power of the torque motor (a function of $n_{m}$ and the external load $F$ ) and $\eta_{m}$ represents the working efficiency of the torque motor.

From Eqs. (12)-(18), the working heat $Q$ of the full-aperture lapping turntable is finally

$$
Q=P_{i n}\left(n_{m}, F\right)\left(1-\eta_{m}\right)+1.047 \times 10^{-4} n_{m}\left(M_{0}+M_{1}\right) .
$$

From Eq. (19), when the turntable-shaft structure and the external load are constant, $Q$ depends only on the rotational speed $n_{m}$ of the torque motor. Therefore, $Q$ can also be expressed as

$$
Q=f\left(n_{m}\right)
$$

where $f$ is a mapping from $n_{m}$ to $Q$.

\subsection{Regression analysis of the flexural deformation of the lapping-pad surface shape}

The flexural deformation of the lapping-pad surface shape is a type of thermal deformation, controlled by the RCLPS system and the working heat $Q$. According to the standard literature, an accurate thermal deformation model for machine tools can be established by regression analysis based on measured experimental data [23].

The structure of the turntable-shaft system based on the RCLPS system is symmetrical along the rotary axis $C$; the flexural deformation of the lapping-pad surface shape is therefore a symmetrical curved surface with rotational axis $C$, and can be represented by a radial deformation curve $w_{c}$. The $n^{\text {th }}$ degree polynomial regression equation for the radial deformation curve is

$$
w_{c}(\rho)=\sum_{i=0}^{n} \theta_{i} \rho^{n}+\varepsilon
$$

where $\rho$ is the distance between the sampling point and the $C$ axis along the radial direction, $c$ is the turntable shaft system state, $\left\{\theta_{0}, \theta_{1}, \ldots, \theta_{n}\right\}$ is the undetermined coefficient, $\varepsilon$ is the calculation error, and $\varepsilon \sim N\left(0, \sigma^{2}\right)$. 
Let $x_{1}, x_{2}, \ldots, x_{n}$ be defined as $x_{1}=\rho, x_{2}=\rho^{2}, \ldots, x_{n}=\rho^{n}$. Then, Eq. (21) can be transformed into

$$
w_{c}(\rho)=\theta_{0}+\sum_{i=1}^{n} \theta_{i} x_{i}+\varepsilon
$$

a multiple-linear-regression equation. The set of sampling points $W$ is defined as $\left\{\left(w_{c}\left(\rho_{i}\right), \rho_{i}\right) \mid i=1,2,3, \ldots, k\right\}$, where $i$ and $k$ represent the $i^{t h}$ sampling point and the number of sampling points, respectively; $\rho_{i}$ and $w_{c}\left(\rho_{i}\right)$ are the distance and the deformation for the $i^{\text {th }}$ sampling point. Bringing $W$ into Eq. (22), the following equations can be obtained:

$$
\left\{\begin{array}{c}
w_{c}\left(\rho_{1}\right)=\theta_{0}+\sum_{i=1}^{n} \theta_{i} x_{i}+\varepsilon=\theta_{0}+\theta_{1} \rho_{1}+\theta_{2} \rho_{1}^{2}+\ldots+\theta_{n} \rho_{1}^{n}+\varepsilon \\
w_{c}\left(\rho_{2}\right)=\theta_{0}+\sum_{i=1}^{n} \theta_{i} x_{i}+\varepsilon=\theta_{0}+\theta_{1} \rho_{2}+\theta_{2} \rho_{2}^{2}+\ldots+\theta_{n} \rho_{2}^{n}+\varepsilon \\
\ldots \\
w_{c}\left(\rho_{k}\right)=\theta_{0}+\sum_{i=1}^{n} \theta_{i} x_{i}+\varepsilon=\theta_{0}+\theta_{1} \rho_{n}+\theta_{2} \rho_{n}^{2}+\ldots+\theta_{n} \rho_{n}^{n}+\varepsilon
\end{array}\right.
$$

If we introduce $X, Y$, and $B$, defined as $X=\left[\begin{array}{cccc}1 & \rho_{1} & \ldots & \rho_{1} \\ 1 & \rho_{2} & \ldots & \rho_{2}{ }^{n} \\ \ldots & \ldots & \ldots & \ldots \\ 1 & \rho_{k} & \ldots & \rho_{k}{ }^{n}\end{array}\right], Y=\left[\begin{array}{c}w_{c}\left(\rho_{1}\right) \\ w_{c}\left(\rho_{2}\right) \\ \ldots \\ w_{c}\left(\rho_{k}\right)\end{array}\right], B=\left[\begin{array}{c}\theta_{0} \\ \theta_{1} \\ \ldots \\ \theta_{n}\end{array}\right]$, Eq. becomes $\mathrm{Y}=\mathrm{BX}+\varepsilon$. Finally, the regression equation is obtained by maximum likelihood estimation of $B$ :

$$
\mathrm{B}=\left(\mathrm{X}^{\prime} \mathrm{X}\right)^{-1} \mathrm{X}^{\prime} \mathrm{Y}
$$

The undetermined coefficients $\left\{\theta_{0}, \theta_{1}, \ldots, \theta_{n}\right\}$ are given by Eq. (24), and the regression equation for the radial flexure-deformation curve $w_{c}$ can be obtained by inserting them into Eq. (21).

\section{Experimental program}

\subsection{Measurement of the flexural deformation of the lapping-pad surface shape}

The experiments were designed to measure the flexural deformation of the lapping-pad surface controlled by the RCLPS system (control parameter $t_{c}$ ) and the working heat $Q$ (control parameter $n_{m}$ ) of the turntable-shaft system. A laser sensor (Model: LK-H020, Keyence Corporation) was used to measure the flexural deformation at different values of $t_{c}$ and $n_{m}$. The experimental apparatus included the lapping turntable based on the RCPLS system, dressing shaft, and laser sensor, as shown in Fig. 4. The laser sensor, which was fixed on the dressing shaft, was controlled to move linearly along the $X$-axis. The turntable 
shaft was driven by a torque motor with $360^{\circ}$ rotation along the $C$ axis. The flexural-deformation curve of the lapping-pad surface was measured in the radial direction; this is sufficient to describe the overall deformation of the lapping-pad surface.

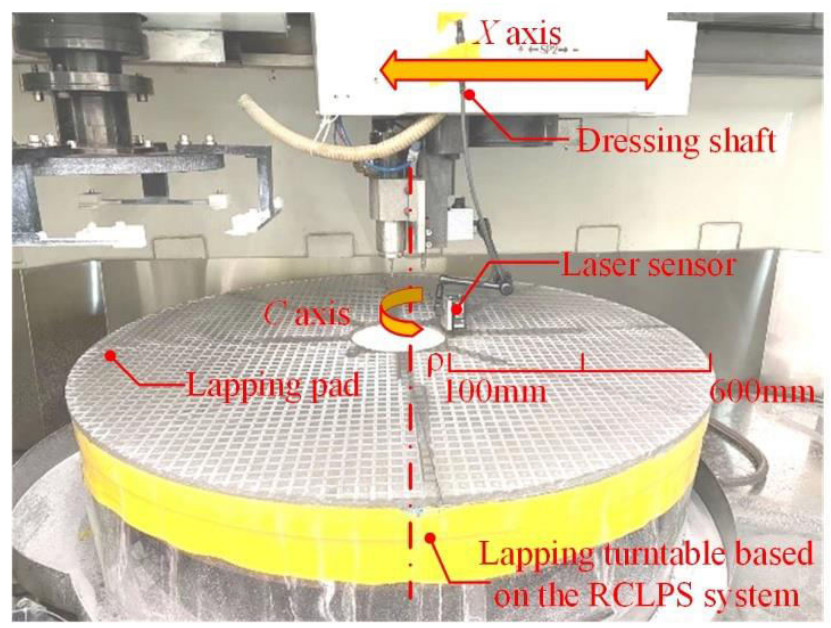

Fig. 4 Measurement of the flexural-deformation curve for the lapping-pad surface

According to Eqs. (10) and (20), the temperature $t_{c}$ of the high-precision water chiller and rotational speed $n_{m}$ of the torque motor are the control parameters for the RCLPS system, and the working heat $Q$ is the control parameter for the turntable. The flexural-deformation curve of the lapping-surface shape is measured by varying these parameters.

\subsection{Process optimization experiment based on parameter $t_{c}$}

The control parameter $t_{c}$ of the RCLPS system can be adjusted in real time during the lapping process; therefore, the parameter $t_{c}$ can be used for process optimization. The influence of parameter $t_{c}$ on the process performance of the plane optical component was verified by the experiments described below.

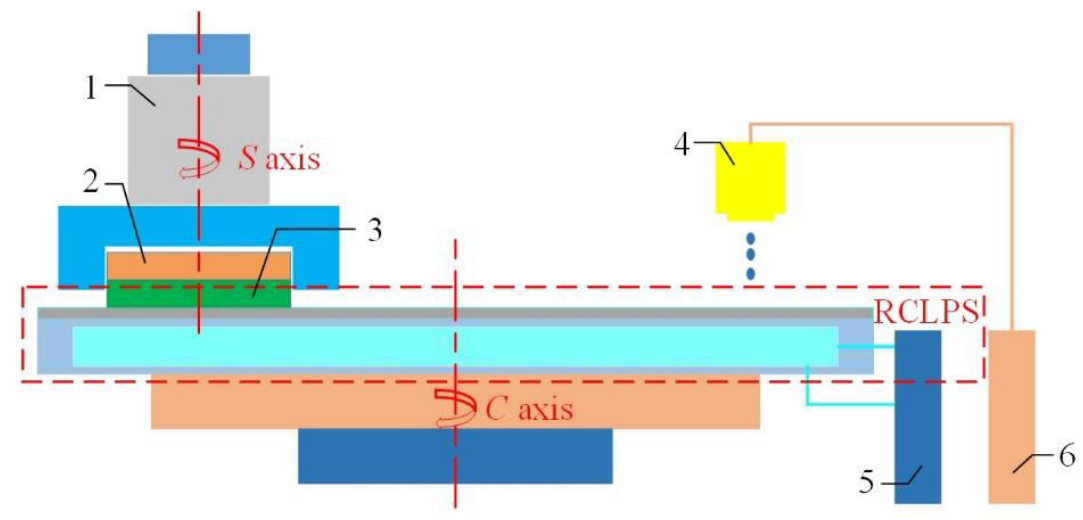

Fig. 5 Structure of the lapping process system: 1. Workpiece shaft; 2. Weights; 3. Plane optical components; 4. Third-stage filter; 5. High-precision water chiller for the RCLPS system; 6. High-precision water chiller for the slurry

The control parameter $t_{c}$ of the RCLPS system, the rotation speed $n_{m}$ of the lapping turntable, and the lapping pressure $P$ were set as the process parameters for the process-optimization experiments. The process parameters $t_{c}, n_{m}$, and $P$ are adjusted using precision water chiller 1 , torque motor, and weights, 
respectively. The experimental scheme and settings are shown in Fig. 5 and Table 1, respectively.

Table 1 Parameter settings of process-optimization experiment

\begin{tabular}{cccccc}
\hline name & Lapping pad & Component type & $\begin{array}{c}\text { Component } \\
\text { volume }\end{array}$ & Slurry & $\begin{array}{c}\text { Environment and } \\
\text { slurry temperature }\end{array}$ \\
\hline \multirow{2}{*}{ value } & Metal-tin pad & Fused silica & $\pi 140 \times 40 \mathrm{~mm}^{3}$ & $\mathrm{Al}_{2} \mathrm{O}_{3}$ & $21 \pm 0.5{ }^{\circ} \mathrm{C}$ \\
\end{tabular}

Using the process parameters $t_{c}, n_{m}$, and $P$, three-factor and three-level process-optimization experiments were designed, as shown in Table 2. To improve the efficiency of the experiment, the orthogonal-experiment method was adopted to design a combination of each group of experiments. The $\mathrm{L}_{9}\left(3^{4}\right)$ orthogonal table proposed by Taguchi [24]. was used to arrange each group of experiments. Material-removal rate (MRR) and surface-change uniformity (SCU) were selected to evaluate the results of each set of experiments.

Table 2 Factors and levels of optimization experiments

\begin{tabular}{cccc}
\hline \multirow{2}{*}{ Levels } & \multicolumn{3}{c}{ Factors } \\
\cline { 2 - 4 } & $t_{c}$ & $n_{m}$ & $P$ \\
\hline 1 & $16^{\circ} \mathrm{C}\left(t_{c} 1\right)$ & $8 \mathrm{rpm}\left(n_{m} 1\right)$ & $1 \mathrm{kpa}(P 1)$ \\
2 & $18^{\circ} \mathrm{C}\left(t_{c} 2\right)$ & $4 \mathrm{rpm}\left(n_{m} 2\right)$ & $2 \mathrm{kpa}(P 2)$ \\
3 & $20^{\circ} \mathrm{C}\left(t_{c} 3\right)$ & $12 \mathrm{rpm}\left(n_{m} 3\right)$ & $4 \mathrm{kpa}(P 3)$ \\
\hline
\end{tabular}

\section{Results, discussion, and analysis}

\subsection{The change rate of the flexural deformation with time}

For two typical conditions $\left(t_{c}=19{ }^{\circ} \mathrm{C}\right.$ or $n_{m}=100 \mathrm{rpm}$ ), the change rates (per $20 \mathrm{~min}$ ) of the flexure-deformation curve for the lapping-pad surface are shown in Figures 6(a) and (b) respectively. According to Fig. 6(a), when the RCLPS system worked independently $\left(\right.$ at $t_{c}=19^{\circ} \mathrm{C}$ ), the change rate from 0 to 20 min was the largest; with the increase in working time, it gradually decreased, becoming close to 0 when the working time was greater than $60 \mathrm{~min}$. When the turntable ran independently $\left(n_{m}=100 \mathrm{rpm}\right)$, similar laws for the change rate were obtained, as shown in Figure 6(b): the change rate from 0 to 20 min was the largest, and, when the working time was greater than $60 \mathrm{~min}$, the change rate (60-80 $\mathrm{min})$ tended to be stable (the flexural-deformation curve no longer changed significantly). 


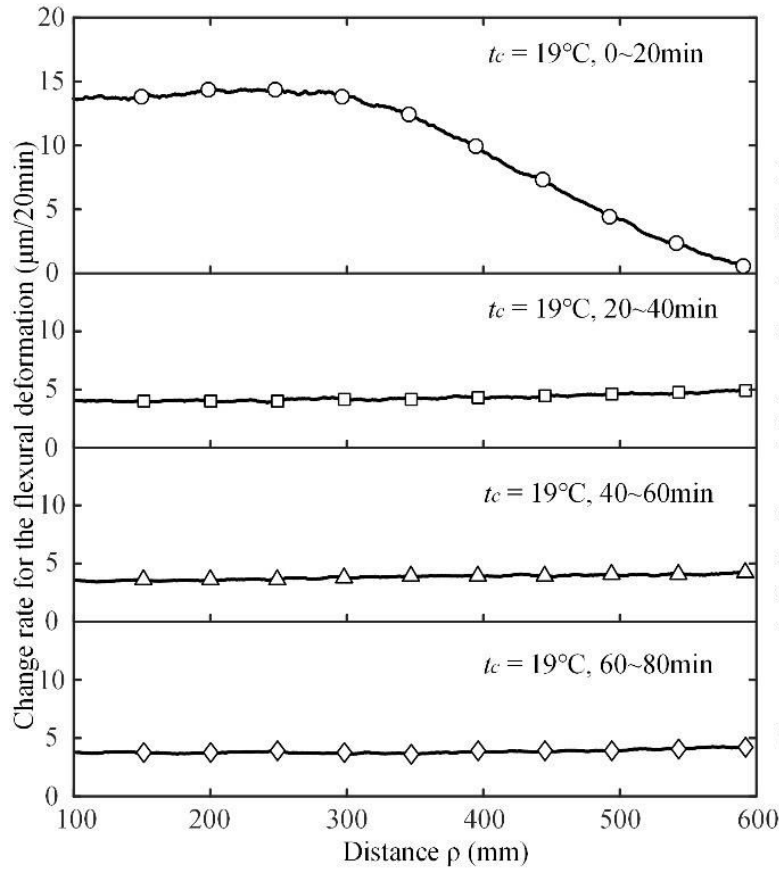

(a)

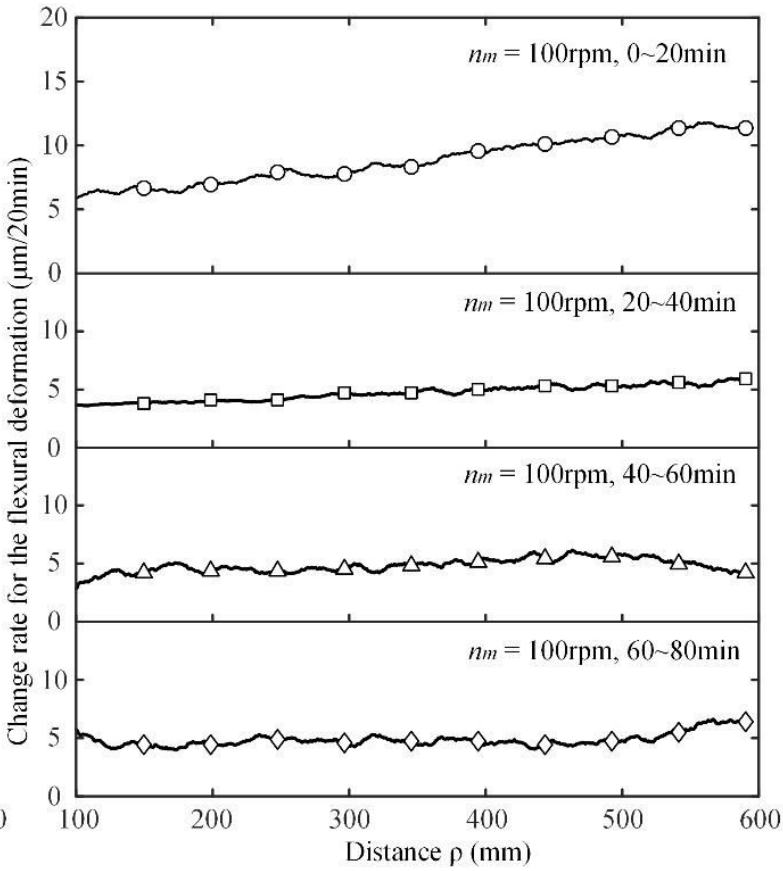

(b)

Fig 6 The change rate of the lapping-pad surface radial flexural-deformation curve for two typical conditions: (a)

RCLPS system works independently (at $t_{c}=19^{\circ} \mathrm{C}$ ); (b) Turntable runs independently (at $n_{m}=100 \mathrm{rpm}$ )

In summary, the flexural deformation of the lapping-pad surface reached a stable state when the working time of the RCLPS system was greater than $60 \mathrm{~min}$. The thermal-deformation error for the metal lapping disc also tended to be stable when the working time for the turntable exceeded $60 \mathrm{~min}$.

\subsection{The thermal-deformation error for the lapping-pad surface caused by the working heat $Q$}

After the turntable ran independently for $70 \mathrm{~min}\left(n_{m}=100 \mathrm{rpm}\right)$, the temperature-field distribution for the turntable shaft system was detected by the thermal video system (Figure 7(c)). At different rotational speeds for the turntable $\left(n_{m}=20 \mathrm{rpm}, 60 \mathrm{rpm}\right.$ and $\left.100 \mathrm{rpm}\right)$, the thermal-deformation error of the lapping-pad surface was measured, as shown in Figures 7(a) and 7(b).
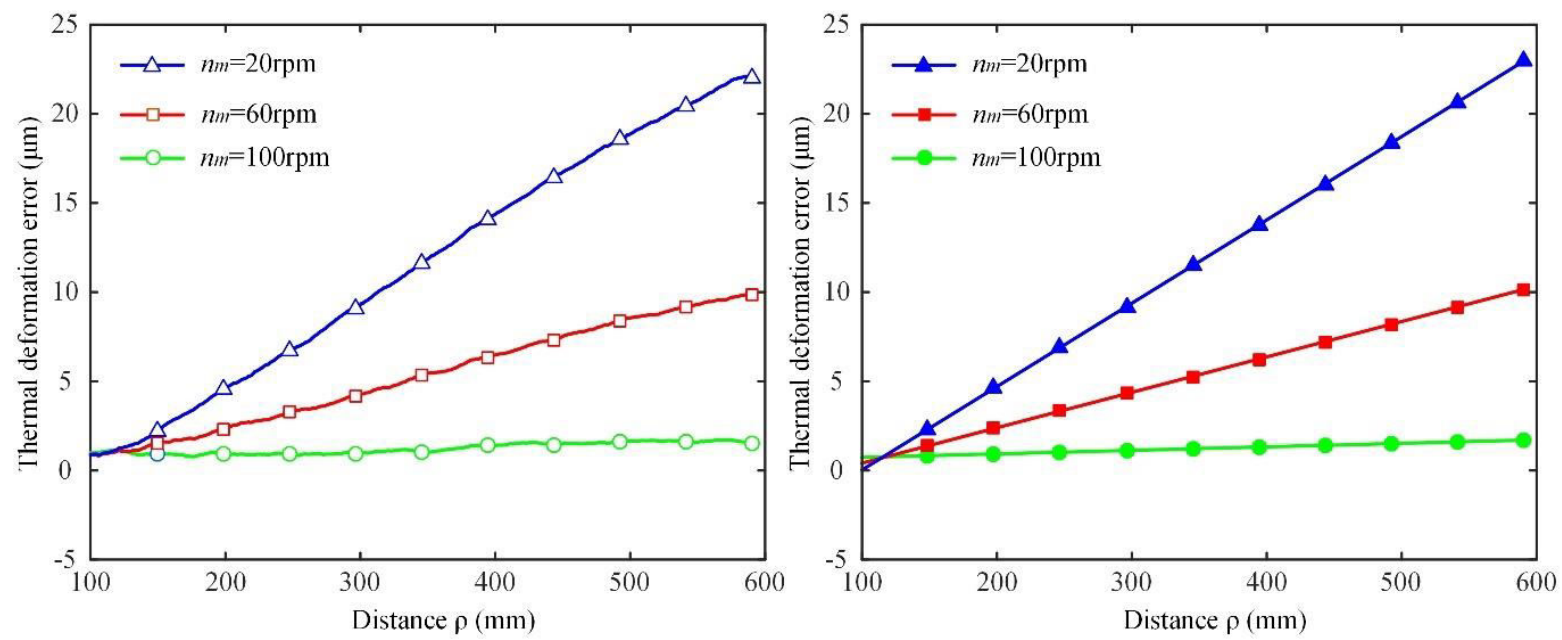


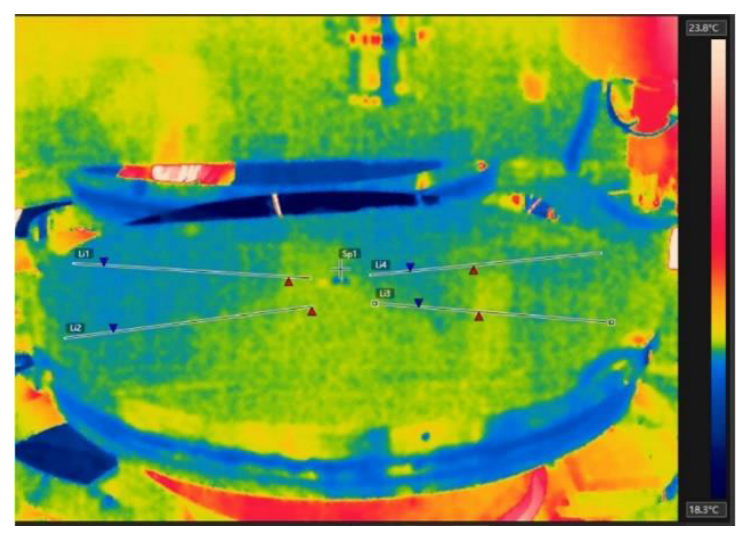

(c)

Fig. 7 Thermal imaging of the turntable and thermal-deformation error of the lapping-pad surface after the turntable ran independently for $70 \mathrm{~min}$ : (a) Thermal-deformation-error curves for the lapping-pad surface at 20, 60, and 100 rpm; (b) Regression analysis for the thermal-deformation-error curves; (c) Thermal image of the turntable-shaft system at $100 \mathrm{rpm}$

From Fig. 7(c), the temperature at the bottom of the turntable (close to the torque motors and bearings) increased because of the working heat $Q$. Therefore, thermal-deformation errors for the lapping-pad surface were generated, as shown in Figures 7(a) and 7(b). As the rotational speed $n_{m}$ increased, so did the thermal-deformation errors. At $n_{m}=20 \mathrm{rpm}$, the thermal-deformation-error curve was approximately a horizontal line, which means that there were no thermal-deformation errors of the lapping-pad surface. The largest slope of the thermal-deformation error curve was at $100 \mathrm{rpm}$, which means that the thermal-deformation errors there were the largest and $w_{\max }=20 \mu \mathrm{m}$. According to Eq. (24), the linear regression equations of the curves of the thermal-deformation errors are

$$
w^{T D E}(\rho)= \begin{cases}0.0029 \rho+7.704 & n_{m}=20 \mathrm{rpm} \\ 0.0198 \rho+6.020 & n_{m}=60 \mathrm{rpm} \\ 0.0466 \rho+3.336 & n_{m}=100 \mathrm{rpm}\end{cases}
$$

where $w^{T D E}(\rho)$ is called the thermal-deformation error model for the lapping pad.

In the mass production of plane optical elements, both the turning dressing of the lapping-pad surface and the lapping process of the plane element are important. The rotation speed of the turntable is usually set to $n_{m} \leq 20 \mathrm{rpm}$ and $n_{m}=100 \mathrm{rpm}$ during lapping and turning correction, respectively. Thus, in the lapping process for the optical elements, the thermal-deformation error of the lapping-pad surface due to the working heat $Q$ is approximately 0 , which can be uncompensated. By contrast, in the turning dressing for the lapping pad $\left(n_{m}=100 \mathrm{rpm}\right)$, the thermal-deformation error generates a curve with a large slope, which must compensated; the compensation equations are shown in Eq. (25).

\subsection{The dressing and the thermal error compensation of the lapping-pad surface based on the RCLPS system}

For the lapping-pad-surface shape, real-time dressing during the lapping process and thermal-error 
compensation during turning dressing are realized by the RCLPS system, as shown in Figures 8 and 9.

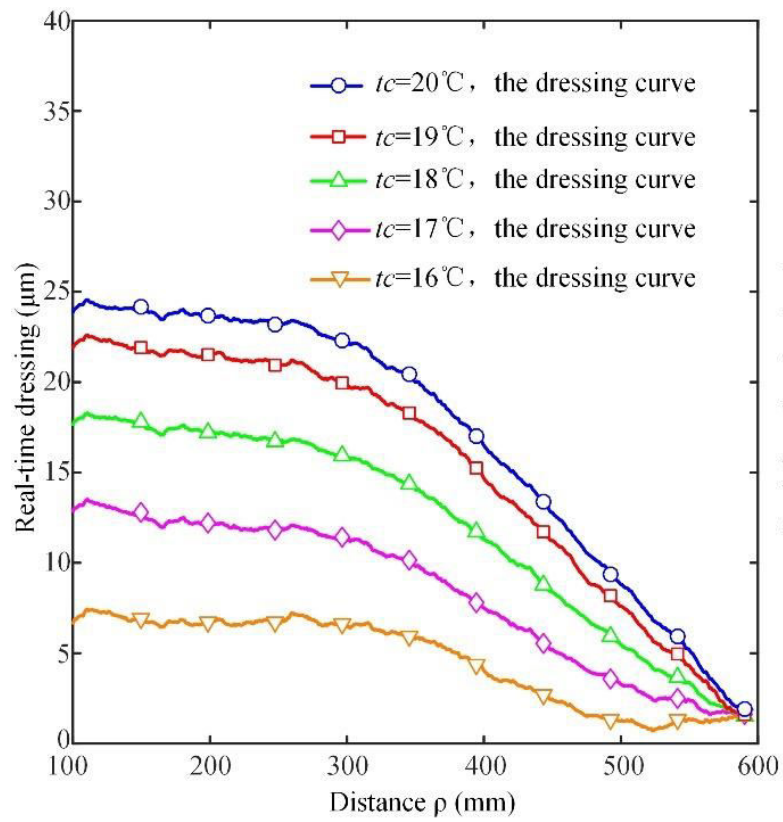

(a)

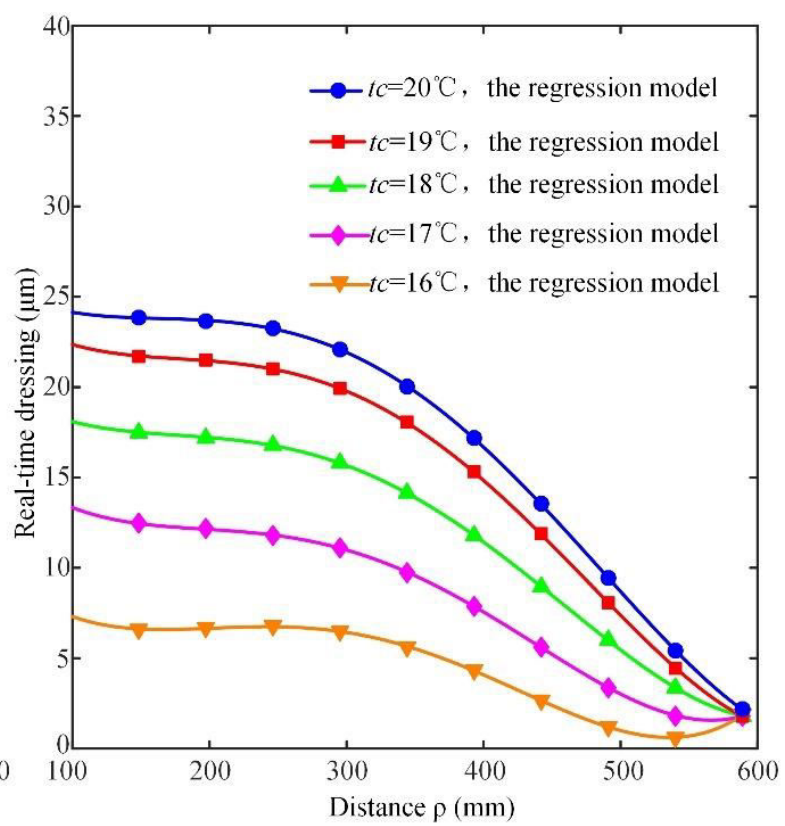

(b)

Fig. 8 Real-time dressing curves during lapping process at $20 \mathrm{rpm}$ for various water-chiller temperatures $t_{c}$ : (a) Real-time dressing curves for the lapping-pad surface at $16,17,18,19$ and $20{ }^{\circ} \mathrm{C}$; (b) Regression analysis for real-time dressing curves

From Fig. 8, during the lapping process $\left(n_{m} \leq 20 \mathrm{rpm}\right)$, the flexural-deformation curves (real-time dressing curves ) of the lapping-pad surface were controlled by the parameter $t_{c}$ for the RCLPS system. The real-time dressing curve at $t_{c}=16{ }^{\circ} \mathrm{C}$ was closer to a horizontal straight line than the other curves, which means that the flexural deformation for the lapping-pad surface shape was the smallest. From $\rho=100-300$ $\mathrm{mm}, w$ was maximal, with $w_{\max }=5 \mu \mathrm{m}$. From $\rho=300-500 \mathrm{~mm}, w$ decreased as the radial distance $\rho$ increased. The flexural deformation remained unchanged from 500-590 mm. Also, as the control parameter $t_{c}$ increased (from $16{ }^{\circ} \mathrm{C}$ to $20^{\circ} \mathrm{C}$ ), $w$ increased as well. The maximum flexural deformation (at $\rho=100 \mathrm{~mm}$ ) increased from $5 \mu \mathrm{m}$ to $20 \mu \mathrm{m}$. Finally, in general, from $t_{c}=16{ }^{\circ} \mathrm{C}-20{ }^{\circ} \mathrm{C}$, the convex deformation of the lapping-pad surface was caused by the RCLPS system, and the degree of convex deformation increased with an increase in the control parameter $t_{c}$. According to Eq. (24), the polynomial regression equations of the real-time dressing curves are

$$
w^{R D}(\rho)=\left\{\begin{array}{lll}
(1.63 e-9) \rho^{4}-(2.05 e-6) \rho^{3}+(8.47 e-4) \rho^{2}-(1.41 e-1) \rho+14.96 & t_{c}=16^{\circ} \mathrm{C} \\
(1.47 e-9) \rho^{4}-(1.86 e-6) \rho^{3}+(7.55 e-4) \rho^{2}-(1.30 e-1) \rho+20.63 & t_{c}=17^{\circ} \mathrm{C} \\
(1.24 e-9) \rho^{4}-(1.57 e-6) \rho^{3}+(6.03 e-4) \rho^{2}-(9.8 e-2) \rho+23.43 & t_{c}=18^{\circ} \mathrm{C} \\
(1.35 e-9) \rho^{4}-(1.76 e-6) \rho^{3}+(6.83 e-4) \rho^{2}-(1.10 e-1) \rho+28.35 & t_{c}=19^{\circ} \mathrm{C} \\
(1.20 e-9) \rho^{4}-(1.57 e-6) \rho^{3}+(5.75 e-4) \rho^{2}-(8.52 e-2) \rho+28.49 & t_{c}=20^{\circ} \mathrm{C}
\end{array}\right.
$$

where $w^{R D}(\rho)$ is the real-time dressing curve prediction model for the RCLPS system.

During turning dressing $\left(n_{m}=100 \mathrm{rpm}\right)$, the thermal-deformation error curves compensated by the RCLPS system are described, as shown in Fig.9. In general, as the control parameter $t_{c}$ increased, the 
thermal-deformation error first decreased and then increased. At $t_{c}=19{ }^{\circ} \mathrm{C}$, the smallest thermal-deformation error was obtained, and $w_{\max }$ fell from $25 \mu \mathrm{m}$ (without compensation) to $3 \mu \mathrm{m}\left(t_{c}=\right.$ $\left.19^{\circ} \mathrm{C}\right)$. Therefore, the thermal-deformation error was effectively compensated for by the RCLPS system.

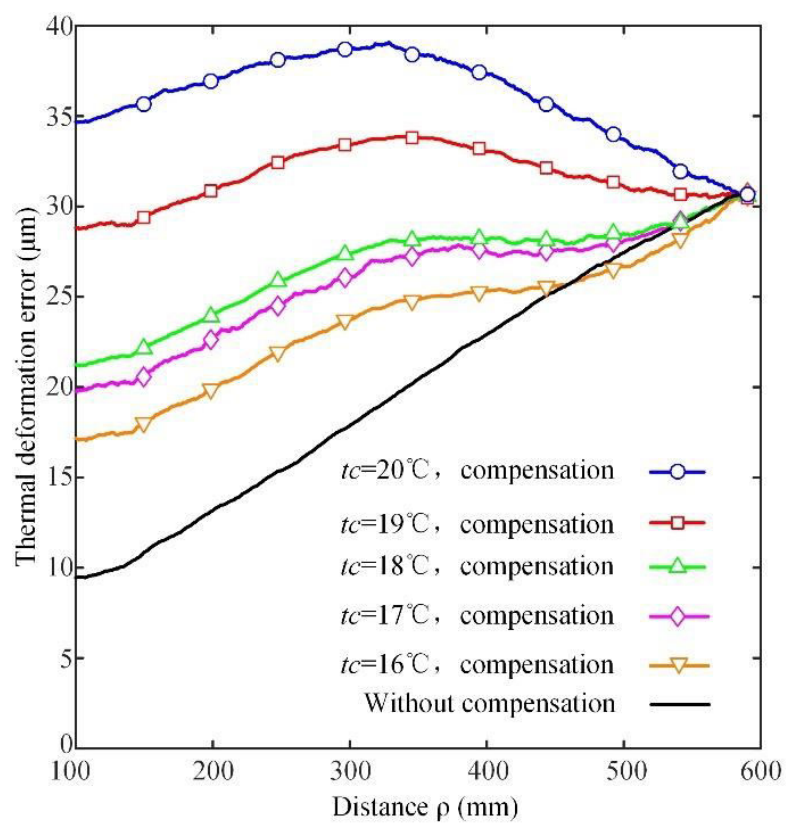

Fig. 9 Thermal-deformation error curves compensated by the RCLPS system during turning dressing of the lapping-pad surface shape at $100 \mathrm{rpm}$.

In summary, during the lapping process $\left(n_{m} \leq 20 \mathrm{rpm}\right)$, real-time dressing of the lapping-pad surface shape was realized by the RCLPS system. By adjusting the control parameter $t_{c}$ of the RCLPS system, different real-time dressing curves could be obtained. During turning dressing $\left(n_{m}=100 \mathrm{rpm}\right)$, the thermal-deformation error for the working heat $Q$ was compensated by the RCLPS system. At $t_{c}=19{ }^{\circ} \mathrm{C}$, the smallest value of the thermal-deformation error of the lapping-pad surface-shape occurred.

\subsection{Process-parameter-optimization based on control parameter $t_{c}$}

The combination and measurement results of the orthogonal experiments are listed in Table 3. The evaluation indicators MRR and SCU are defined by [25]

$$
\begin{aligned}
& \operatorname{MRR}_{i}=\frac{m_{i}-m_{i-1}}{\rho_{m} \cdot s_{m} \cdot t_{m}}, \quad i=1,2,3, \ldots, n \\
& \mathrm{SCU}_{i}=\sqrt{\frac{\sum_{j=1}^{l}\left[z_{i}\left(x_{j}, y_{j}\right)-z_{i-1}\left(x_{j}, y_{j}\right)\right]^{2}}{n}}, i=1,2,3, \ldots, n,
\end{aligned}
$$

where $i$ and $n$ is the $i^{t h}$ experiment and the number of experiments repectively, $m_{i}$ is the quality of the component after the $i^{\text {th }}$ experiment, $m_{0}$ is the initial weight of the component, $\rho_{m}$ is the material density of the component, $s_{m}$ is the process area of the component, $t_{m}$ represents the process time of 
each experiment, and $z_{i}\left(x_{j}, y_{j}\right)$ represents the relative height of the $j^{\text {th }}$ point $\left(x_{j}, y_{j}\right)$ after the $i^{\text {th }}$ experiment, $l$ is the number of the measured points.

Table $3 L_{9}\left(3^{4}\right)$ Orthogonal experimental design and results

\begin{tabular}{cccccc}
\hline No & & Factor & & $\begin{array}{c}\text { MRR } \\
x_{i}^{M R R} n m / h\end{array}$ & $\begin{array}{c}S C R \\
x_{i}^{S C R} n m\end{array}$ \\
\cline { 2 - 3 } & $\boldsymbol{t}_{\boldsymbol{c}}$ & $\boldsymbol{n}_{\boldsymbol{m}}$ & $\boldsymbol{P}$ & 49.16 & 27.00 \\
2 & $t_{c} 1$ & $n_{m} 1$ & $P 1$ & 132.74 & 33.40 \\
3 & $t_{c} 1$ & $n_{m} 2$ & $P 2$ & 245.82 & 44.00 \\
4 & $t_{c} 1$ & $n_{m} 3$ & $P 3$ & 147.49 & 33.00 \\
5 & $t_{c} 2$ & $n_{m} 1$ & $P 2$ & 271.39 & 42.00 \\
6 & $t_{c} 2$ & $n_{m} 2$ & $P 3$ & 98.33 & 37.02 \\
7 & $t_{c} 2$ & $n_{m} 3$ & $P 1$ & 255.65 & 51.30 \\
8 & $t_{c} 3$ & $n_{m} 1$ & $P 3$ & 33.43 & 18.80 \\
9 & $t_{c} 3$ & $n_{m} 2$ & $P 1$ & 393.31 & 56.90 \\
\hline
\end{tabular}

Table 4 Range analysis results

\begin{tabular}{cccc}
\hline \multirow{2}{*}{ MRR range analysis } & \multicolumn{3}{c}{ Factors } \\
\cline { 2 - 4 } & \multicolumn{1}{c}{$\boldsymbol{t}_{\boldsymbol{c}}$} & $\boldsymbol{n}_{\boldsymbol{m}}$ & $\boldsymbol{P}$ \\
\hline $\bar{K}_{1}$ & $142 \mathrm{~nm} / \mathrm{h}$ & $151 \mathrm{~nm} / \mathrm{h}$ & $60 \mathrm{~nm} / \mathrm{h}$ \\
$\bar{K}_{2}$ & $172 \mathrm{~nm} / \mathrm{h}$ & $146 \mathrm{~nm} / \mathrm{h}$ & $225 \mathrm{~nm} / \mathrm{h}$ \\
$\bar{K}_{3}$ & $227 \mathrm{~nm} / \mathrm{h}$ & $246 \mathrm{~nm} / \mathrm{h}$ & $258 \mathrm{~nm} / \mathrm{h}$ \\
$R_{M R R}$ & $85 \mathrm{~nm} / \mathrm{h}$ & $100 \mathrm{~nm} / \mathrm{h}$ & $197 \mathrm{~nm} / \mathrm{h}$ \\
\hline \multirow{2}{*}{ SCU range analysis } & & Factors & \\
\cline { 2 - 4 } & $\boldsymbol{T}$ & $\boldsymbol{S}$ & $28 \mathrm{~nm}$ \\
$\bar{K}_{1}$ & $35 \mathrm{~nm}$ & $37 \mathrm{~nm}$ & $41 \mathrm{~nm}$ \\
$\bar{K}_{2}$ & $37 \mathrm{~nm}$ & $31 \mathrm{~nm}$ & $46 \mathrm{~nm}$ \\
$\bar{K}_{3}$ & $42 \mathrm{~nm}$ & $46 \mathrm{~nm}$ & $18 \mathrm{~nm}$ \\
$R_{S C U}$ & $7 \mathrm{~nm}$ & $15 \mathrm{~nm}$ & \\
\hline
\end{tabular}

The results of the range analysis are shown in Figure 10 and Table 4. MRR and SCU were affected by the 
control parameter $t_{c}$ of the RCLPS system, as shown in Figures 10(a) and (b): they increased with an increase in $t_{c}$. $P$ was the factor with the most influence on MRR and SCU, and $t_{c}$ the factor with the least. The optimal combinations for MRR and SCU, analyzed by the range method, were $t_{c} 3 n_{m} 2 P 3$ and $t_{c} 1 n_{m} 1 P 1$, respectively. The experimental results for the optimal combinations are presented in Table 5, which shows that the optimal combination for the different optimization targets obtains the best results in their respective optimization indicators.

Table 5 Optimal parameter combinations

\begin{tabular}{ccc}
\hline Target for optimization: & MRR & SCU \\
\hline combination & $t_{c} 3 n_{m} 2 P 3$ & $t_{c} 1 n_{m} 1 P 1$ \\
$M R R$ & $442 \mathrm{~nm} / \mathrm{h}$ & $40 \mathrm{~nm} / \mathrm{h}$ \\
$S C U$ & $58 \mathrm{~nm}$ & $10 \mathrm{~nm}$ \\
\hline
\end{tabular}

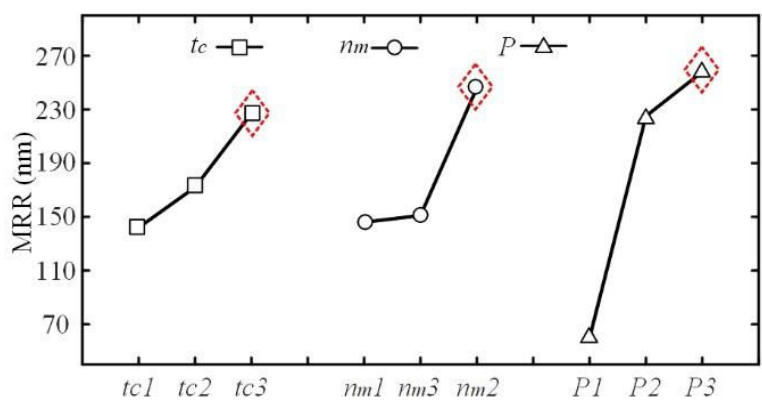

(a)

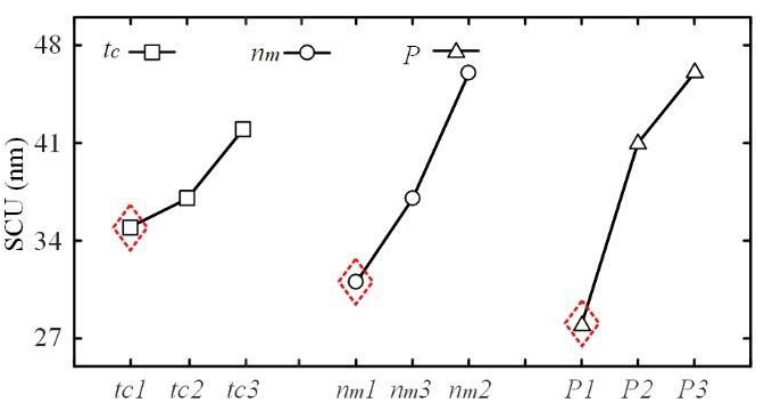

(b)

Fig. 10 Range analysis results for (a) MRR and (b) SCU . Red diamonds indicate optimal combinations. $t_{c}$ : control parameter of the RCLPS system; $n_{m}$ : rotation speed; $P$ : lapping pressure

Based on the above experiment and analysis, the control parameter $t_{c}$ of the RCLPS system can be set as a process parameter to optimize the MRR and SCU.

\section{Conclusion}

The RCLPS system based on the bimetal thermal-deformation effect was proposed to realize real-time dressing of the lapping-pad surface shape. The working heat $Q$ of the full-aperture turntable was analyzed. Experimental and theoretical analyses of the RCLPS system led to the following conclusions:

1) The flexure deformation and thermal-deformation error for the lapping-pad surface shape were caused by the RCLPS system and the working heat $Q$, which is stable at working times $\geq 60 \mathrm{~min}$.

2) During turning dressing of the lapping pad $\left(n_{m}=100 \mathrm{rpm}\right)$, the thermal-deformation error is caused by the working heat $Q$, which should be compensated. During the lapping process for optical elements $\left(n_{m} \leq\right.$ $20 \mathrm{rpm}$ ), the thermal-deformation error due to $Q$ is small, and it can be left uncompensated

3) In turning dressing $\left(n_{m}=100 \mathrm{rpm}\right)$, the thermal-deformation error caused by the working heat $Q$ can be compensated by the RCLPS system, which is smallest at $t_{c}=19^{\circ} \mathrm{C}$. In the lapping process $\left(n_{m} \leqslant 20 \mathrm{rpm}\right)$, the real-time dressing of the lapping-pad surface shape can be controlled by the control parameter $t_{c}$ of the RCLPS system $\left(t_{c}=16^{\circ} \mathrm{C}-20{ }^{\circ} \mathrm{C}\right)$. 
4) The control parameter $t_{c}$, which is a process parameter, can be used for process optimization. Both the MRR and SCU are affected by the control parameter $t_{c}$. The best results for MRR and SCU were obtained by $t_{c} 3 n_{m} 2 P 3$ and $t_{c} 1 n_{m} 1 P 1$, respectively.

\section{Acknowledgments}

Not applicable.

\section{Ethical Approval}

Not applicable.

\section{Consent to Participate}

Not applicable.

\section{Consent to Publish}

Not applicable.

\section{Authors Contributions}

Conceptualization, L.Z., H.Z. and R.X.; methodology, L.Z., H.Z. and R.X.; formal analysis, L.Z. and H.W.; investigation, L.Z., M.C., M.Z. and S.Z.; writing-original draft preparation, L.Z.; writing-review and editing, H.Z., H.W., and S.Z.

\section{Funding}

This work is supported by Science Challenge Project of China (No. TZ2016006-0501-03); National Defense Science and Industry Administration basic Product Innovation Program vehicle power research special project (No. DEDPZF); Complete equipment project for ultra-precision processing of optical materials (No. TC190JED-207; No. TC190JED-210)

\section{Competing Interests}

The authors declare that there is no conflict of interest regarding the publication of this paper

\section{Availability of data and materials}

Not applicable.

\section{REFERENCES}

[1] Yaguo Li, Qinghua Zhang, Jian Wang, Qiao Xu, Hui Ye (2017) 1.6 Precision Grinding, Lapping, Polishing, and Post-Processing of Optical Glass. Reference. Comprehensive Materials Finishing. M. S. J. Hashmi. Oxford, Elsevier:154-170.

[2] Urara Satake, Toshiyuki Enomoto, Teppei Miyagawa, Takuya Ohsumi, Hidenori Nakagawa, Katsuhiro Funabashi (2019) Stabilization of Removal Rate in Small Tool Polishing of Glass Lenses. Int. J. Autom. Tech. 13(2): 221-229.

[3] Rongyan Sun, Atsunori Nozoe, Junji Nagahashi, Kenta Arima, Kentaro Kawai, Kazuya Yamamura (2021) Novel highly-efficient and dress-free polishing technique with plasma-assisted surface modification and 
dressing. Precis. Eng. 72: 224-236

[4] Jun Xu, Yongwei Zhu, Nannan Zhu, Jianbin Wang, Jun Li (2014) Self-Conditioning Mechanism of Hydrophilic Fixed Abrasive Pad. Nanotechnol. Precis. Eng. 12(6): 429-434.

[5] X. X. Ban, H. Y. Zhao, S. J. Zhao, R. Q. Xie, Y. W. Gu, X. L. Zhu, D. F. Liao, L. Li, Z. D. Jiang (2019) Effect of geometry error on accuracy of large-diameter pads used for CMP dressing. Int. J. Adv. Manuf. Technol. 100:1505-1520.

[7] D. Liao, Z. Yuan, C. Tang, R. Xie, X. Chen (2013) Mid-Spatial Frequency Error (PSD-2) of optics induced during CCOS and full-aperture polishing. Journal of the J. Eur. Opt. Soc.-Rapid Publ. 8:13031

[8] M. Y. Tsai, J. C. Sung (2009) Dressing Behaviors of PCD Conditioners on CMP Polishing Pads. Adv. Mater. Res. 76-78: 201-206.

[9] Hyuk-Min Kim, Gun-Ho Park, Young-Gil Seo, Deog-Ju Moon, Byoung-Jun Cho, Jin-Goo Park (2015). Comparison between sapphire lapping processes using 2-body and 3-body modes as a function of diamond abrasive size. Wear 332-333: 794-799.

[10] Hyunseop Lee (2020). Simulation on Polishing Pad Wear in CMP Conditioning with Split Conditioner Disk. 2020 International Conference on Electronics, Information, and Communication (ICEIC) pp. 1-2.

[11] Quoc-Phong Pham, Chao-Chang A. Chen (2017) Study on pad cutting rate and surface roughness in diamond dressing process. Int. J. Precis. Eng. Manuf. 18(12): 1683-1691.

[12] Chao-Chang A. Chen, Quoc-Phong Pham (2017) Study on diamond dressing for non-uniformity of pad surface topography in CMP process, Int. J. Adv. Manuf. Technol. 91:3573-3582.

[13] Fei Ma, Hui Huang (2020) Experimental research on the biological in-process dressing (BID) of Cu-Co matrix diamond tools. J. Clean Prod. 275:124070.

[14] Yuang-Cherng Chiou, Rong-Tsong Lee,Chang-Li Yau (2007) A novel method of composite electroplating on lap in lapping process. Int. J. Mach. Tools Manuf. 47(2):361-367.

[15] Ri Pan, Yuhang Tang, Bo Zhong, Dongju Chen, Zhenzhong Wang, Jinwei Fan, Chunyan Zhang (2019) Qualitative Motion Control Optimization of the Pad Dressing Process for Bonnet Tool, IEEE-ASME Trans. Mechatron. 24(3): 1141-1152.

[16] M.Y. Tsai, P.H. Li, J.C. Sung (2012) Organic diamond disk versus brazed diamond disk for dressing a chemical-mechanical polishing pad. Diam. Relat. Mat. 23: 144-149.

[17] Taekyung Lee, Hyoungjae Kim, Sangjik Lee, Chuljin Park, Doyeon Kim, Haedo Jeong (2017) Self-dressing effect using a fixed abrasive platen for single-sided lapping of sapphire substrate. J. Mech. Sci. Technol. 31(12): 5649-5655.

[18] Ruiqing Xie, Shijie Zhao, Defeng Liao, Xianhua Chen, Jian Wang, Qiao Xu, Huiying Zhao, Zhuangde Jiang (2019) Surface Characteristics of Polished YAG Laser Crystal. Cryst. Res. Technol. 54(5):1800274.

[19] Zhifeng Zhou, Yudian Fan (1996) A Study of Thermal Stress in Thin Films. Chin. J. Vac. Sci. Technol. 16(5): 347-354. https://doi.org/10.13922/j.cnki.cjovst.1996.05.007

[20] Zhilun Xu (2006) Elasticity. Higher Education Press, Beijing

[21] Chi Ma, Jialan Liu, Shilong Wang (2020) Thermal error compensation of linear axis with fixed-fixed installation. Int. J. Mech. Sci. 175:105531. 
[22] Jialan Liu, Chi Ma, Hongquan Gui, Shilong Wang (2021) Thermally-induced error compensation of spindle system based on long short term memory neural networks. Appl. Soft. Comput. 102:107094.

[23] T.Narendra Reddy, V. Shanmugaraj, Prakash Vinod, S.Gopi Krishna (2020) Real-time Thermal Error Compensation Strategy for Precision Machine tools. Materials Today: Proceedings 22:2386-2396.

[24] Ilhan Asiltürk, Harun Akkus (2011) Determining the effect of cutting parameters on surface roughness in hard turning using the Taguchi method. Measurement 44(9):1697-1704

[25] Mingchen Cao, Huiying Zhao, Ruiqing Xie, Shijie Zhao, Lingyu Zhao, Jinfeng Bai (2021) Enhancing parameter design or full-aperture rapid planar polishing based on fuzzy optimization. Appl. Optics 60(17): 5049 5055. 
